\title{
Effects of Supplementation with Saccharomyces cerevisiae and Its Fermentation Products on Production Performance and Its Mechanism in Perinatal Dairy Cows
}

\author{
Xueyan Lin, Ke Li, Lin Ju, Xu Hao, Yue Jiang, Qiuling Hou, Zhiyong Hu, Yun Wang, \\ Zhonghua Wang*
}

College of Animal Science, Shandong Agricultural University, Tai'an, China

Email: linxueyan@sdau.edu.cn, *zhwang@sdau.edu.cn

How to cite this paper: Lin, X.Y., Li, K., Ju, L., Hao, X., Jiang, Y., Hou, Q.L., Hu, Z.Y., Wang, Y. and Wang, Z.H. (2021) Effects of Supplementation with Saccharomyces cerevisiae and Its Fermentation Products on Production Performance and Its Mechanism in Perinatal Dairy Cows. Advances in Bioscience and Biotechnology, 12, 193-212.

https://doi.org/10.4236/abb.2021.127013

Received: June 2, 2021

Accepted: July 12, 2021

Published: July 15, 2021

Copyright $\odot 2021$ by author(s) and Scientific Research Publishing Inc. This work is licensed under the Creative Commons Attribution International License (CC BY 4.0).

http://creativecommons.org/licenses/by/4.0/ (c) (i) Open Access

\begin{abstract}
The aim of this study was to investigate the effects of Saccharomyces cerevisiae and its fermentation products on performance, blood hormone levels and rumen floral composition in peripartum dairy cows. Sixty perinatal cows were selected and allocated to two groups according to parity and expected date of delivery. Each group was supplemented with Saccharomyces cerevisiae and its fermentation product 0 or $100 \mathrm{~g}$. The results showed that Saccharomyces cerevisiae and its fermentation products could significantly increase the feed intake of peripartum dairy cows $(\mathrm{P}<0.01)$, increase the lactose content after 21 days postpartum $(\mathrm{P}<0.01)$, and tend to increase milk production $(\mathrm{P}=0.052)$. There was no significant effect on other milk components, the apparent digestibility of nutrients. There was a tendency to increase milk production and reduce the number of somatic cells in milk; increase blood levels of glucagon $(\mathrm{P}<0.01)$ and $\beta$-hydroxybutyrate $(\mathrm{P}=0.01)$, reducing the insulin content $(\mathrm{P}=0.02)$. Saccharomyces cerevisiae reduced the abundance of rumen microbes in peripartum dairy cows but had no effect on rumen microbial diversity. Compared with the control group, the supplemented group showed reductions in the abundance of genera Bacillus $(\mathrm{P}=0.03)$, Butyrivibrio $(\mathrm{P}=0.01)$, Denitrobacterium $(\mathrm{P}=0.01)$, and Mogibacterium $(\mathrm{P}<0.01)$, Porphyromonas $(\mathrm{P}=0.05)$, Saccharofermentans $(\mathrm{P}<0.01)$, Sphaerochaeta $(\mathrm{P}$ $=0.02)$, Streptococcus $(\mathrm{P}=0.04)$ and other genera. There were significant increase in the content of Acidaminococcus $(\mathrm{P}=0.03)$, Allisonella $(\mathrm{P}<0.01)$, Bulleidia $(\mathrm{P}<0.01)$, Corynebacterium $(\mathrm{P}=0.01)$, Dialister $(\mathrm{P}<0.01)$, Faecalibacterium $(\mathrm{P}=0.02)$, Faekalitalea $(\mathrm{P}=0.03)$, Fibrobacter $(\mathrm{P}=0.04)$, Flavobacterium $(\mathrm{P}=0.03)$, Kandleria $(\mathrm{P}<0.01)$, Paraprevotella $(\mathrm{P}<0.01)$, Pyramidobacter $(\mathrm{P}=0.05)$, Roseburia $(\mathrm{P}<0.01)$, Succinivibrio $(\mathrm{P}<0.01)$ and
\end{abstract}


other genera. The main metabolic pathways such as tryptophan metabolism and steroid hormone biosynthesis in perinatal dairy cows were determined for Saccharomyces cerevisiae and its fermentation products.

\section{Keywords}

Saccharomyces cerevisiae, Perinatal Period, Dairy Cows, Production

Performance, Mechanism

\section{Introduction}

The perinatal period is also called the transition period. It is the period from the late pregnancy of the cow to the beginning of lactation. It generally refers to the cow 21 days perinatal period to 21 days postpartum period [1]. It is characterized by the need for a large amount of energy for lactation. However, the negative energy balance caused by the low intake of feed causes the body to mobilize a lot of body fat, and excessive accumulation of fat around the liver leads to the occurrence of fatty liver. At the same time, fat metabolism causes an increase in ketone body content to cause ketosis. This seriously affects the normal reproductive and digestive physiology of the cows, disturbs the metabolism of nutrients, and regulates neuroendocrine, which in turn leads to nutritional metabolic disorders and even illness. These will reduce the performance of the cow in the next lactation cycle. Saccharomyces cerevisiae can stimulate the rumen microorganisms of ruminants through its metabolites, regulate the micro-ecological balance, and promote the growth of beneficial bacteria to achieve the purpose of improving production performance. Saccharomyces cerevisiae also improves rumen fermentation, improves feed digestibility and utilization efficiency, microbial quantity and vigor, and enhances immunity. The rumen flora affects host production performance. The feed conversion efficiency of beef cattle is closely related to the rumen microbial community structure, which is reflected in many studies [2] [3]. Jami suggested that milk fat production showed a significant positive correlation with F/B (proportion of Copticella and Bacteroides) and a significant negative correlation with $P$. vivax under Bacteroides [4].

The addition of Saccharomyces cerevisiae to dairy cow diets can increase the dry matter intake of lactating dairy cows, milk yield [5], increase the relative viability of cellulase, and promote the degradation of cellulose in feeds in the rumen. Saccharomyces cerevisiae reduces rumen $\mathrm{pH}$ [5] and ammonia nitrogen concentration. At the same time, studies have shown that yeast can promote the proliferation of other rumen microorganisms [6], reducing the number of somatic cells in milk, and can adsorb toxins, pathogenic bacteria and degradation of aflatoxins etc. However, studies have also indicated that $S$. cerevisiae has no effect on dry matter intake in peripartum dairy cows [7], or can only increasing prenatal feed intake has no effect on postpartum feed intake [8], and has no effect on total digestive dry matter, cell wall composition, and nitrogen digestibili- 
ty.

The purpose of this study was to determine the true effect and mechanism of yeast addition in cows in the perinatal period. The results of this study hope to further determine the role of yeast in the perinatal period of dairy cows, which has important guiding significance for production.

\section{Materials and Methods}

\subsection{Test Animals}

60 perinatal Holstein cows were selected and divided into two groups according to the expected delivery period $(2 \pm 0.69) .30$ heads per group. The trial period began from 21 days before the expected date of delivery of each cow to 21 days after delivery, for a total of about 42 days. Perinatal cows were fed total mixed ration (TMR) at 7:00 and 19:00 daily during the trial. At 7:30 and 19:30, the TMR was fed to the cows in the perinatal period and milked. They were free to eat and drink freely $24 \mathrm{~h}$ a day. Saccharomyces cerevisiae and its ferment were added to the TMR, and the two test groups were fed 0, $100 \mathrm{~g}$ per day. The cows rushed into the perinatal field 21 days before the expected date of delivery, entered the delivery room 7 days before the expected date of delivery, rushed into the care shed 1 day after delivery, and entered the new born cowshed 7 days after delivery.

\subsection{Diet}

Table 1 shows the formulation and composition of the diet.

Table 1. Formulation and composition of the diet.

\begin{tabular}{ccc}
\hline Item & Prepartum & Postpartum \\
\hline Diet ingredients (Kg) & & 18.0 \\
Corn silage & 16.0 & 4.5 \\
Alfalfa hay & & 0.5 \\
Wild-rye hay & 4.0 & 1.6 \\
Cottonseed & & 10.9 \\
Concentrate supplement & 6.0 & 0.2 \\
Fatty powder & & 0.15 \\
Soda & & \\
Nutrients (\%) & & 47.7 \\
DM & 47.8 & 14.3 \\
CP & 11.6 & 3.6 \\
EE & 5.5 & 14.73 \\
NDF & 48.4 & 45.8 \\
ADF & 24.3 & 23.3 \\
Ash & 8.0 & 10.3 \\
Acid insoluble ash & 1.6 & 1.7 \\
NEL & & \\
\hline
\end{tabular}

Note: $\mathrm{NEL}=\mathrm{GE} \times[94.280-61.5730(\mathrm{NDF} / \mathrm{OM})] \times 0.5501-0.395$. 


\subsection{Sample Collection}

The amount of TMR and the amount of remaining material fed per day were recorded. The TMR sample was collected weekly and placed in an electric constant temperature blast dryer at $65^{\circ} \mathrm{C}$ to a constant weight, and pulverized after 24 hours of resurgence. The cows were taken from the rectum before the morning feeding on the 19th, 20th, and 21st days after the cows were placed in the refrigerator, and stored in a refrigerator. The milk production was recorded for three days, and the milk composition was measured after mixing the milk. In the morning of 21 days after the postpartum, the cows took $10 \mathrm{ml}$ of blood from the tail vein, and centrifuged to take the serum for cryopreservation. $100 \mathrm{ml}$ of rumen fluid was taken through the esophagus. After filtering through 4 layers of gauze, some of them were cryopreserved by metaphosphoric acid and the other part was stored in liquid nitrogen. Transfer to a $-80^{\circ} \mathrm{C}$ refrigerator for long-term storage.

\subsection{Determination Method}

The dry matter determination of TMR and fecal samples was carried out according to the method of GB6435-86; the crude protein was determined according to the Kjeldahl method (GB/T 6432-94); the determination of coarse ash was carried out according to the method of GB/T 6438-86; The crude fat content is carried out according to the method of GB/T 6433-2006; the neutral detergent fiber and the acid detergent fiber are carried out according to the method proposed by Van Soest (1991); the acid-insoluble ash is carried out according to the method of GB/T 23742-2009; Calorimeter (6200, PARR) assay; blood hormones and bioactive substances were determined using the Elisa kit.

\subsection{Statistical Analysis}

Data basic processing was performed using Excel 2007 software, and the results were analyzed by one-way ANOVA using SAS 8.2.

\section{Results}

\subsection{Effect of Saccharomyces cerevisiae and Its Fermentation Products on Apparent Digestibility of Nutrients in Perinatal Dairy Cows}

Table 2 shows the apparent digestibility of various nutrients in peripartum dairy cows. By detecting the apparent digestibility of nutrients, we can see that feeding Saccharomyces cerevisiae and its fermentation products during the perinatal period had no significant effect on the apparent digestibility of nutrients in dairy cows, and only increased the digestibility of crude fat $(\mathrm{P}=0.08)$.

\subsection{Effect on Lactation Performance and DMI of Perinatal Dairy Cows}

As shown in Table 3, the probiotic supplementation during the perinatal period 
Table 2. The apparent digestibility of nutrient ingredients.

\begin{tabular}{ccccc}
\hline Apparent digestibility (\%) & Control & Experiment & SEM & P-value \\
\hline CP & 76.19 & 72.06 & 0.02 & 0.16 \\
ADF & 59.11 & 55.52 & 0.02 & 0.42 \\
NDF & 70.65 & 65.08 & 0.01 & 0.17 \\
EE & 81.57 & 86.55 & 0.02 & 0.08 \\
\hline
\end{tabular}

Table 3. Milk performance and DMI of perinatal cows.

\begin{tabular}{ccccc}
\hline Item & Control & Experiment & SEM & P-value \\
\hline DMI (kg/d) & 19.65 & 21.79 & 0.26 & $<0.01$ \\
Milk yield (kg/d) & 33.21 & 36.61 & 1.37 & 0.06 \\
Milk fat rate (\%) & 3.37 & 3.39 & 0.25 & 0.97 \\
Milk fat yield (kg/d) & 1.13 & 1.20 & 0.03 & 0.28 \\
Milk protein rate (\%) & 3.37 & 3.28 & 0.06 & 0.32 \\
Milk protein yield (kg/d) & 1.10 & 1.23 & 0.07 & 0.34 \\
Lactose rate (\%) & 4.69 & 4.88 & 0.03 & $<0.01$ \\
Somatic cell count (ten thousand/ml) & 20.51 & 15.1 & 6.93 & 0.53 \\
\hline
\end{tabular}

significantly increased the DMI $(\mathrm{P}<0.01)$ and the postpartum lactose content $(\mathrm{P}$ $<0.01)$, with a trend of increased milk production $(\mathrm{P}=0.052)$, but had no significant effect on other milk components.

\subsection{Effect of Saccharomyces cerevisiae and Its Fermentation Product on Blood Biochemical Parameters of Perinatal Dairy Cows}

As can be seen from Table 4 , the $\beta$-hydroxybutyric acid content of the test group was significantly increased $(\mathrm{P}=0.01)$. At the same time, the probiotic preparation increased the content of serum glucagon extremely significantly $(\mathrm{P}<0.01)$, significantly reduced insulin content $(\mathrm{P}=0.0225)$, but had no significant effect on other hormones.

\subsection{Effect of Saccharomyces cerevisiae and Its Ferment on Rumen Fatty Acids in Perinatal Dairy Cows}

As can be seen from Table 5, the contents of butyric acid and isovaleric acid in the test group increased significantly $(\mathrm{P}<0.01, \mathrm{P}=0.02)$. At the same time, the valeric acid content decreased significantly.

\subsection{OTU Venn Graph Analysis}

There were 1726 OUTs detected in total from the two groups. The control group (PC) had a total of 1639 OTUs, of which 293 OTUs were unique to the control group. The test group (PE) had a total of 1433 OTUs, of which 87 OTUs were unique to the test group. A total of 1346 OTUs were common to both treatment groups (Figure 1). 
Table 4. Probiotics on blood biochemical indexes of perinatal dairy cows.

\begin{tabular}{ccccc}
\hline Item & Centrol & Experiment & SEM & P-value \\
\hline IGF-1 $(\mu \mathrm{g} / \mathrm{L})$ & 150.89 & 142.36 & 2.26 & 0.06 \\
glucogon $(\mathrm{ng} / \mathrm{L})$ & 63.74 & 69.57 & 1.05 & $<0.01$ \\
insulin $(\mathrm{mu} / \mathrm{L})$ & 11.99 & 10.47 & 0.34 & 0.02 \\
cortisol $(\mu \mathrm{g} / \mathrm{L})$ & 85.95 & 90.66 & 1.54 & 0.13 \\
prolactin $(\mathrm{ng} / \mathrm{L})$ & 740.33 & 761.46 & 9.93 & 0.3 \\
NIFA $(\mu \mathrm{mol} / \mathrm{L})$ & 379.09 & 389.33 & 3.89 & 0.20 \\
$\beta$-hydroxybutyric acid $(\mu \mathrm{mol} / \mathrm{L})$ & 0.63 & 0.71 & 0.01 & 0.01 \\
leptin $(\mu \mathrm{gg} / \mathrm{L})$ & 7.55 & 7.39 & 0.08 & 0.30 \\
growth hormone $(\mu \mathrm{g} / \mathrm{L})$ & 20.51 & 21.28 & 0.31 & 0.72 \\
TNF-a $(\mathrm{ng} / \mathrm{L})$ & 31.63 & 31.28 & 0.62 & 0.79 \\
\hline
\end{tabular}

Table 5. Volatile fatty acids of perinatal dairy cows.

\begin{tabular}{ccccc}
\hline Item & Control & Experiment & SEM & P-value \\
\hline acetic acid (mmol/L) & 52.05 & 48.86 & 1.02 & 0.12 \\
propionic acid (mmol/L) & 16.50 & 17.34 & 0.40 & 0.31 \\
butyric acid (mmol/L) & 6.48 & 10.57 & 0.57 & $<0.01$ \\
isobutyric acid (mmol/L) & 0.52 & 0.56 & 0.01 & 0.07 \\
valeric acid $(\mathrm{mmol} / \mathrm{L})$ & 1.27 & 0.81 & 0.06 & $<0.01$ \\
isovaleric acid $(\mathrm{mmol} / \mathrm{L})$ & 1.09 & 1.36 & 0.06 & 0.02
\end{tabular}

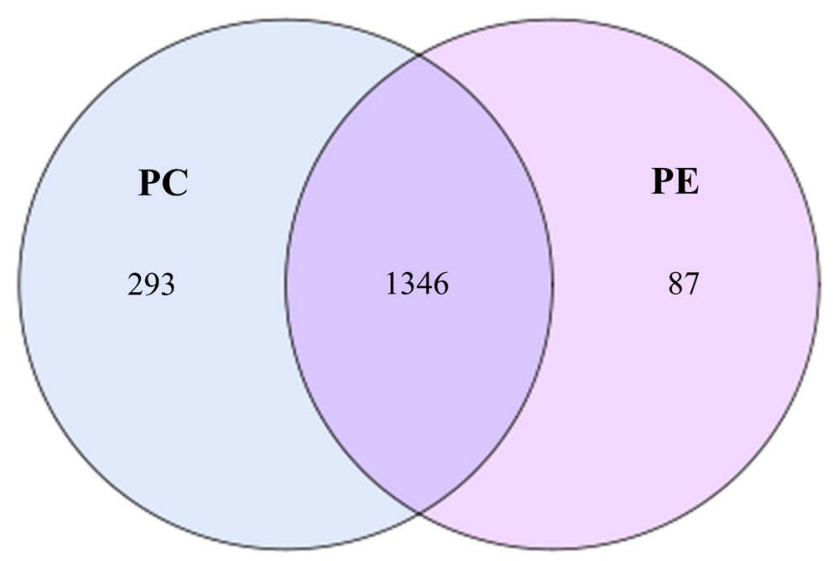

Figure 1. OUT Venn diagram.

\subsection{TU PCA Analysis}

The PCA analysis shows (Figure 2) that the two groups were distributed at two locations. The distance is far and almost without overlap, suggesting that their floral compositions were quite different. Relatively speaking, the data distribution of the red control group (PC) was relatively discrete, indicating that there was a greater deviation in the control group. 


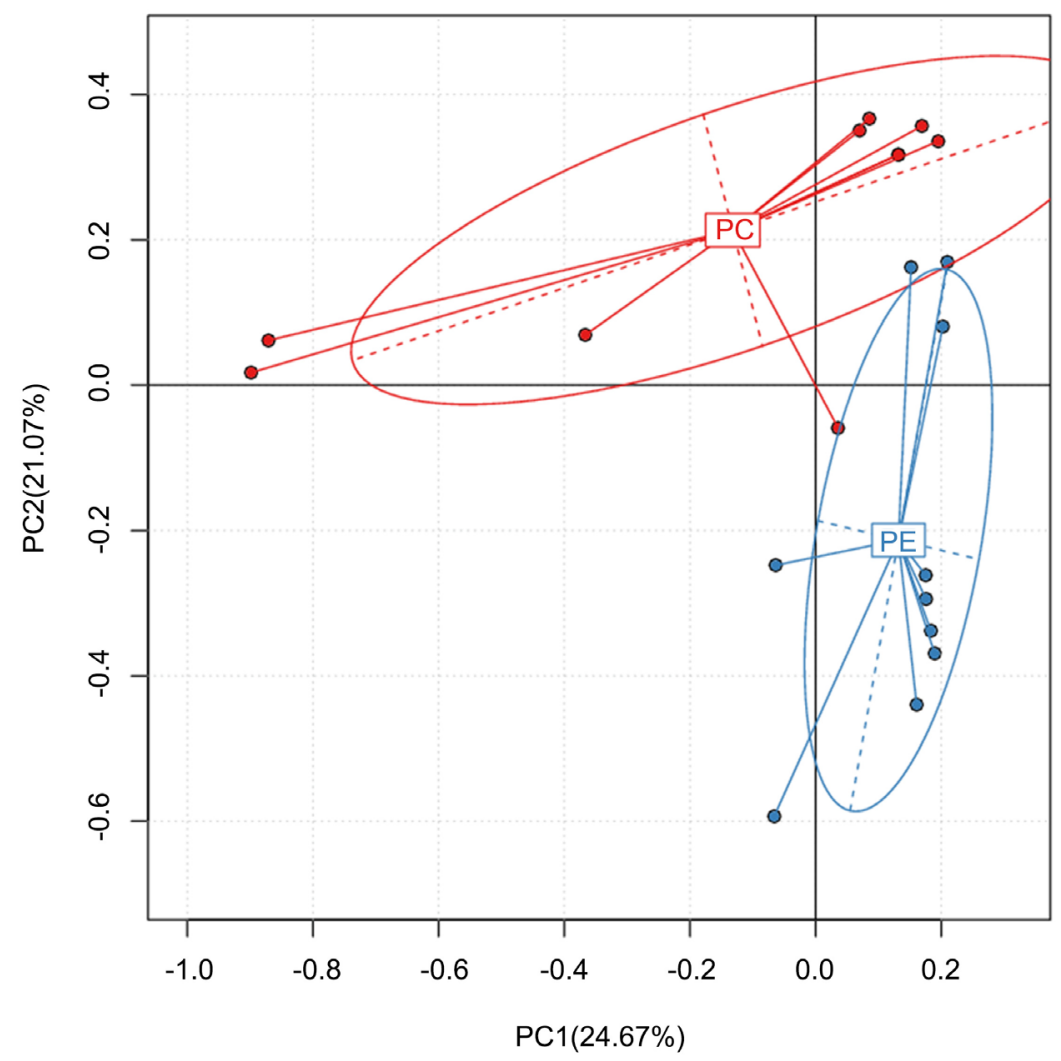

Figure 2. PCA analysis based on OTU abundance (grouped by Description).

\subsection{OTU Rank Curve}

Figure 3 shows that the two treatment groups contained many common OTUs, and this part of OTUs was abundant in each group of samples. Relatively speaking, the samples of the control group were mostly distributed above the test group. Its curve appeared to settle earlier. This suggests that supplementation of Saccharomyces cerevisiae and its fermentation products to perinatal dairy cows may reduce microbial abundance in the rumen.

\subsection{Species Annotation Analysis}

Through the comparison with the database, the OTUs were classified into species and profiling histograms for each sample in several classification levels: phylum, class, order, family, genera and species. The phylum level histogram was shown in column for all species horizontally. Starting from the class level, species with abundance of less than $0.5 \%$ in all samples were combined into the "Others" category.

Figure 4 is a histogram of phyla detected. A total of 14 bacterial phylum were found in this experiment, of which 4 were more than 1\%: Bacteroidetes, Firmicutes, Proteobacteria and Spirochaetes. Hard-walled bacteria in phyla Bacteroides and Proteobacteria accounted for about $90 \%$ of total microorganisms, and 8.4\% (control group) and $11.5 \%$ (test group) of microorganisms were unidentified. Among the 14 phyla, the test group showed significant decreases in the 


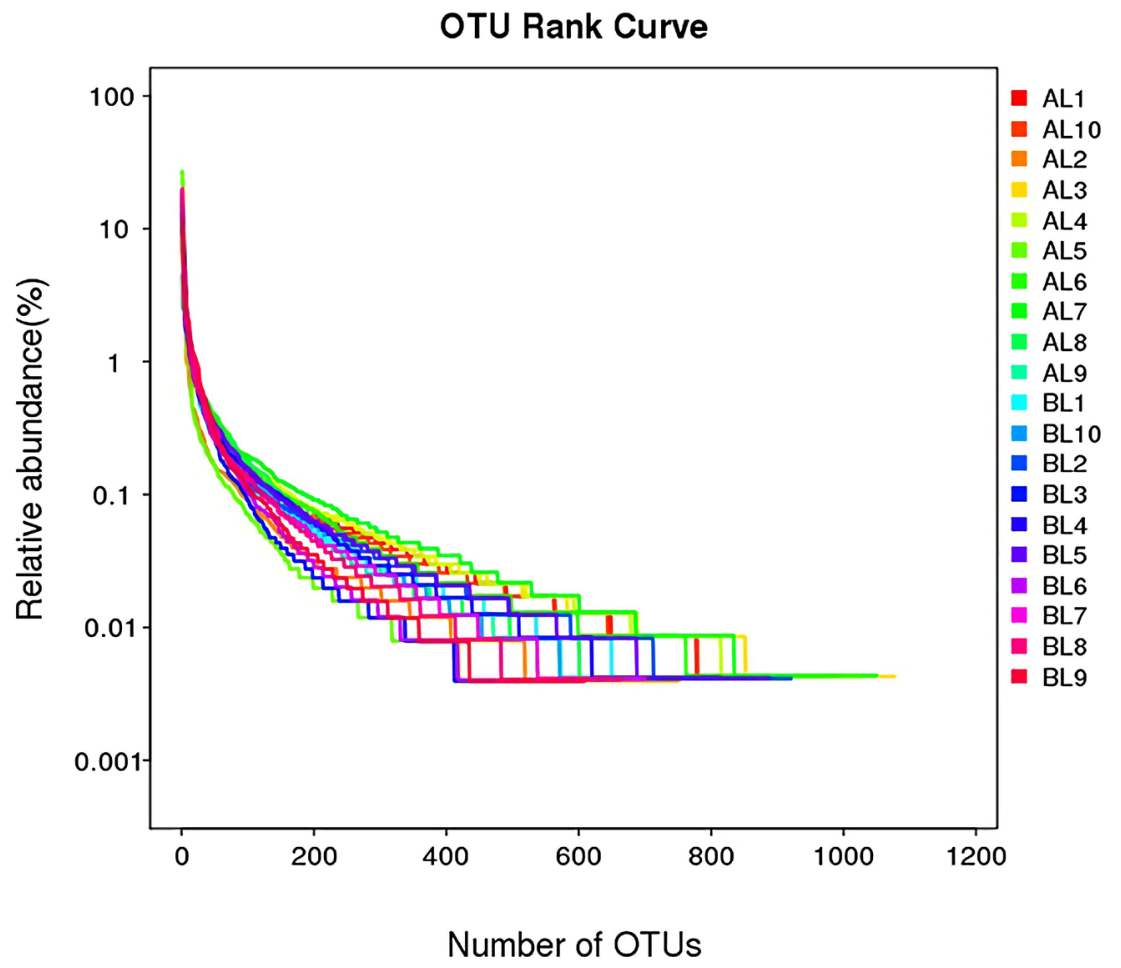

Figure 3. OTU rank curve. Note: The abscissa is the OTU abundance of the sample (high to low) and the ordinate is the OTU abundance.

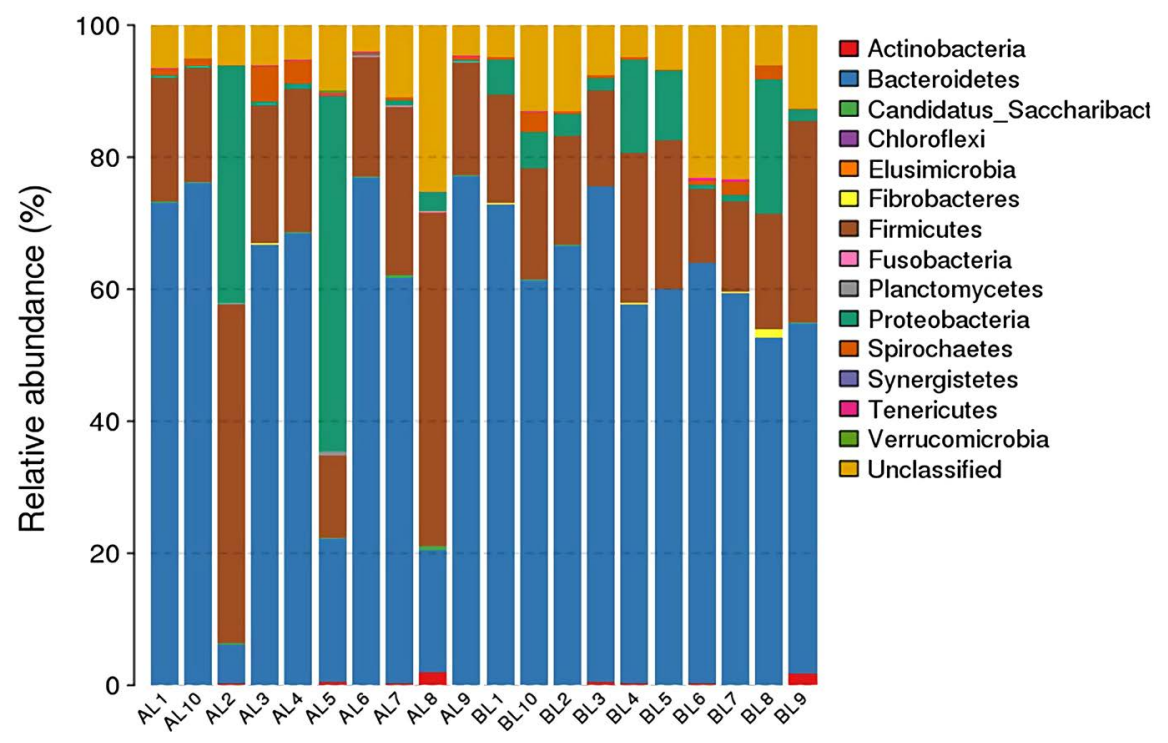

Figure 4. Histogram in the sample at phylum level.

content of Candidatus, Saccharibacteria $(\mathrm{P}=0.01)$, Planctomycetes $(\mathrm{P}<0.01)$, Verrucomicrobia $(\mathrm{P}<0.01)$, while there was a significant increase in the content of Fibrobacteres $(\mathrm{P}=0.01)$.

Figure 5 is a histogram of classes. A total of 24 classes of bacteria were found in the experiment. A total of 5 classes contributed greater than $1 \%$, including Bacteroidia, Clostridium, $\gamma$-proteobacteria, Negativicutes and Spirochaetia. In 


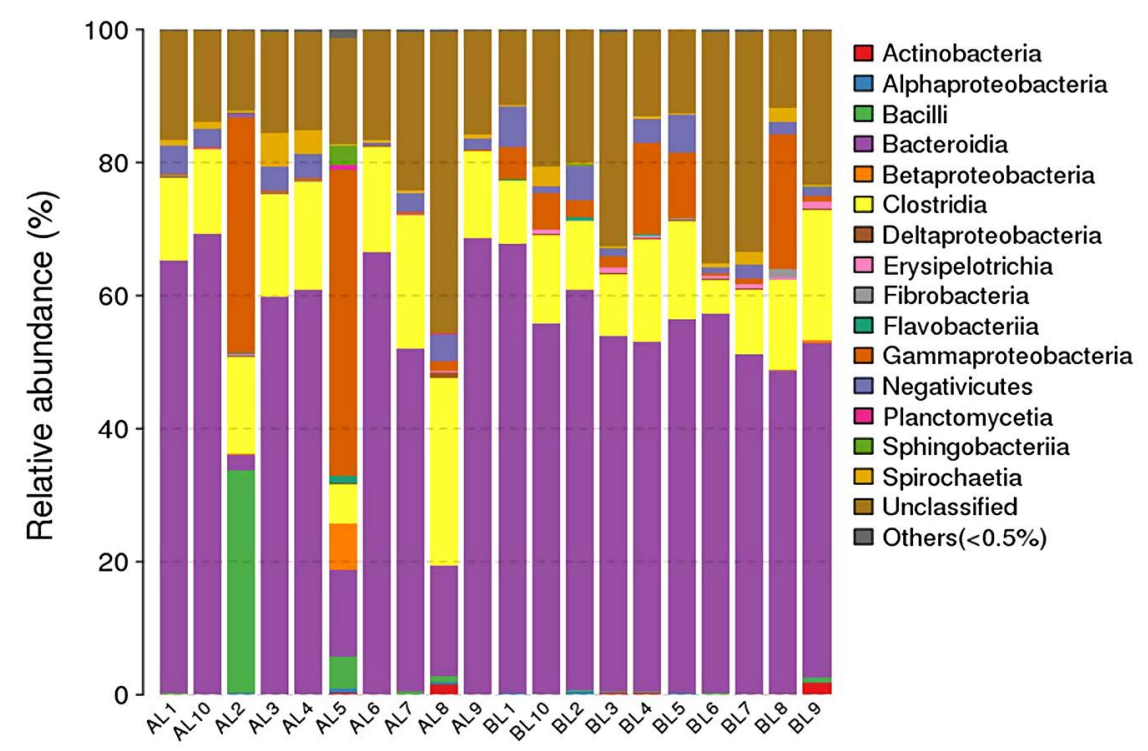

Figure 5. Profile of classes in the microflora.

the 24 classes, the test group showed significant decreases in the contents of Coriobacteriia $(\mathrm{P}<0.01)$, Planctomycetia $(\mathrm{P}<0.01)$, Verrucomicrobiae $(\mathrm{P}<0.01)$, while the content of Erysipelotrichia was significantly increased $(P=0.01)$, and the content of Fibrobacteria was also significantly increased $(P=0.04)$.

Figure 6 is a histogram of orders. A total of 4 orders were detected in this test. A total of 6 classes contributed more than 1\%, including Acidaminococcales, Bacteroidales, Clostridiales, Lactobacillales, Pseudomonadales and Spirochaetales. Among the 4 orders, the test group showed significant decreases in the contents of Bacillales $(\mathrm{P}=0.04)$, Planctomycetales $(\mathrm{P}<0.01)$, Verrucomicrobiales $(\mathrm{P}<$ $0.01)$, while there were significant increases in the content of Aeromonadales ( $\mathrm{P}$ $<0.01)$, Corynebacteriales $(\mathrm{P}=0.01)$, Erysipelotrichales $(\mathrm{P}<0.01)$, Fibrobacterales $(\mathrm{P}=0.04)$, Pasteurellales $(\mathrm{P}=0.02)$, and Veillonellales $(\mathrm{P}<0.01)$.

Figure 7 is a histogram of families. A total of 7 families of bacteria were detected in this experiment. A total of 8 contributed greater than 1\%, including Acidaminococcaceae, Carnobacteriaceae, Lachnospiraceae, Moraxellaceae, Porphyromonadaceae and Prevotellaceae, Ruminococcaceae, Spirochaetaceae. Among the 7 families, significant decreases in the test groups were observed in the contents of Bacillaceae $(\mathrm{P}=0.04)$, Clostridiales_Family_XIII, Incertae_Sedis $(\mathrm{P}<$ $0.01)$, Eggerthellaceae $(P<0.01)$, Ruminococcaceae $(P=0.01)$, Streptococcaceae $(\mathrm{P}=0.04)$, Verrucomicrobia_subdivision_5 $(\mathrm{P}<0.01)$. There were significant increases in the content of Corynebacteriaceae $(\mathrm{P}=0.01)$, Erysipelotrichaceae ( $\mathrm{P}$ $=0.01)$, Fibrobacteraceae $(\mathrm{P}=0.04)$, Pasteurellaceae $(\mathrm{P}=0.02)$, Succinivibrionaceae $(\mathrm{P}<0.01)$, and Veillonellaceae $(\mathrm{P}<0.01)$.

Figure 8 is a histogram of taxa at the genus level. Compared with the control group, the test group showed decreased contents of Bacillus $(\mathrm{P}=0.03)$, Butyrivibrio $(\mathrm{P}=0.01)$, Denitrobacterium $(\mathrm{P}=0.01)$, and Mogibacterium $(\mathrm{P}<0.01)$, Porphyromonas $(\mathrm{P}=0.05)$, Saccharofermentans $(\mathrm{P}<0.01)$, Sphaerochaeta $(\mathrm{P}=$ $0.02)$, Streptococcus $(\mathrm{P}=0.04)$ and other genera. 


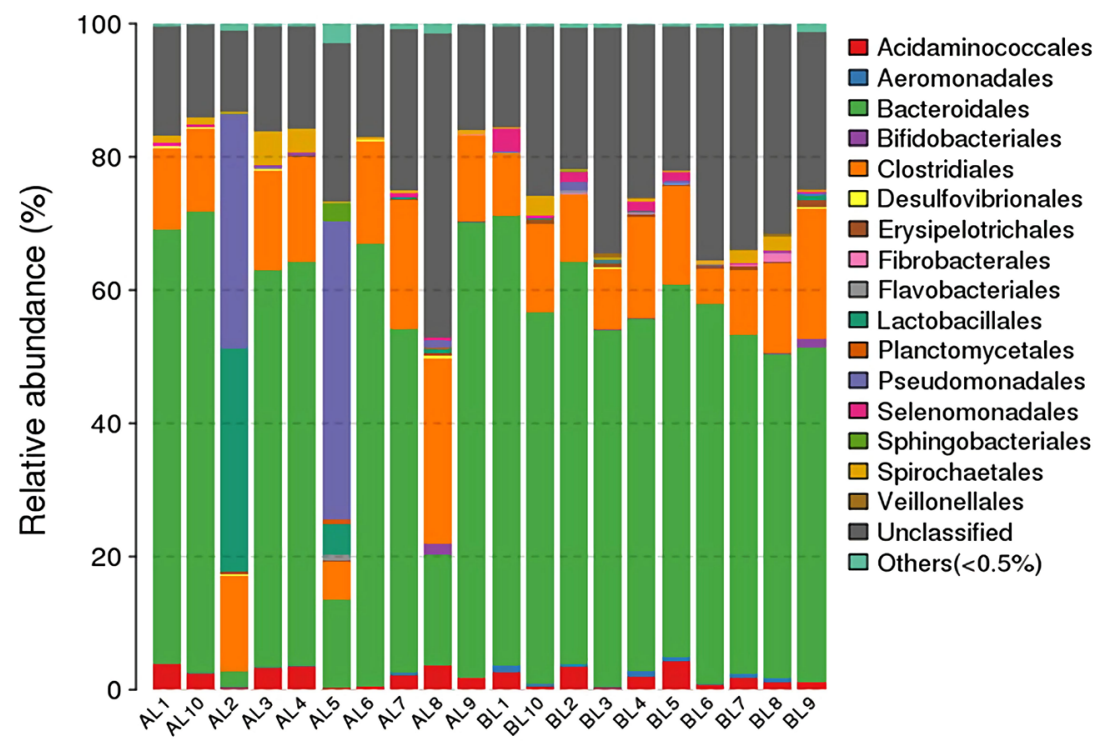

Figure 6. Profile of order level taxa.

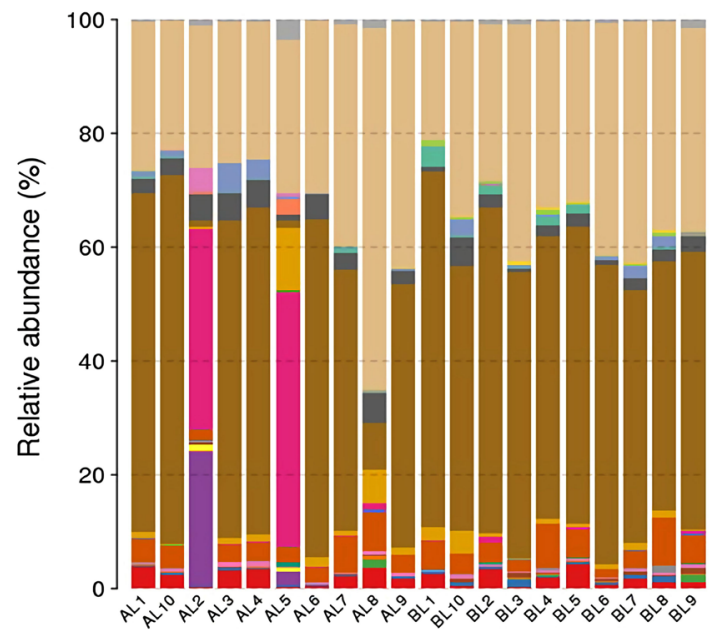

$\square$ Acidaminococcaceae $\square$ Bacteroidaceae $\square$ Bifidobacteriaceae $\square$ Carnobacteriaceae $\square$ Desulfovibrionaceae $\square$ Enterococcaceae $\square$ Erysipelotrichaceae $\square$ Eubacteriaceae $\square$ Fibrobacteraceae $\square$ Flavobacteriaceae $\square$ Lachnospiraceae $\square$ Lactobacillaceae Moraxellaceae

Planctomycetaceae $\square$ Porphyromonadaceae $\square$ Prevotellaceae Ruminococcaceae $\square$ Selenomonadaceae $\square$ Sphingobacteriaceae $\square$ Spirochaetaceae Streptococcaceae $\square$ Succinivibrionaceae $\square$ Veillonellaceae $\square$ Unclassified $\square$ Others $(<0.5 \%)$

Figure 7. Profile of bacterial families in the flora.
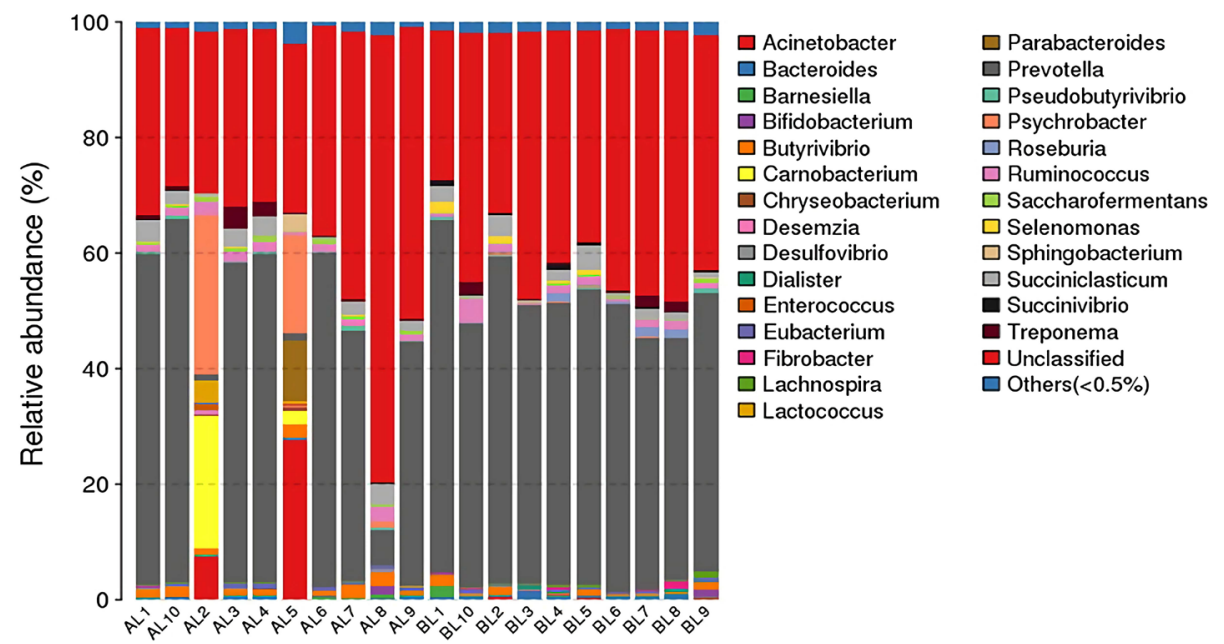

Figure 8. Profile of genus in the microflora. 
There were significant increases in the contents of Acidaminococcus $(\mathrm{P}=$ $0.03)$, Allisonella $(\mathrm{P}<0.01)$, Bulleidia $(\mathrm{P}<0.01)$, Corynebacterium $(\mathrm{P}=0.01)$, Dialister $(\mathrm{P}<0.01)$, Faecalibacterium $(\mathrm{P}=0.02)$, Faekalitalea $(\mathrm{P}=0.03)$, Fibrobacter $(\mathrm{P}=0.04)$, Flavobacterium $(\mathrm{P}=0.03)$, Kandleria $(\mathrm{P}<0.01)$, Paraprevotella $(\mathrm{P}<0.01)$, Pyramidobacter $(\mathrm{P}=0.05)$, Roseburia $(\mathrm{P}<0.01)$, Succinivibrio $(\mathrm{P}<$ $0.01)$ and other genera.

\subsection{Sample Diversity Analysis}

As seen in Figure 9, the observed species (obs), chao values, and ace values of the control group were all above the test group, indicating that the species abundance in the test group was lower than that of the control group. In terms of the Shannon Index and the Simpson Index, there was no significant difference between the two groups. However, it can be seen that the curves of the test group are distributed on both sides of the test group. That is to say, compared with the test group, the control group had significant variations in the species abundance. We can also get the same conclusion from the data and statistics provided in Table 6. Supplementation of probiotics during the perinatal period significantly reduced the abundance of rumen microbes in dairy cows, but had no significant effect on microbial diversity.

\subsection{Effects on Rumen Metabolites and Pathways}

Mass spectrometry data was acquired using a mass spectrometer Xevo G2-XS QTOF (Waters, UK). Statistical analysis of the mass spectrometry data was performed using commercialization software Progenesis QI (version 2.2) (Waters, UK). The metabolite identification was based on the database KEGG. Differentially expressed metabolites were screened using VIP values of the first two principal components of the multivariate PLS-DA model, and in combination with univariate analysis of fold-change and q-value values. Screening conditions were: 1) VIP $\geq 1$; 2) fold-change $\geq 1.2$ or $\leq 0.8333$; 3) q-value $<0.05$. The ions meet these three criteria were considered differential ions. Metabolic pathway analysis was based on the database KEGG (Table 7).

\subsection{Univariate Analysis}

This test was analyzed by $\mathrm{T}$ test and Fold change analysis (FC analysis). In the

Table 6. The Alpha diversity of microbes in perinatal cows.

\begin{tabular}{cccccc}
\hline \#Alpha & Control & SD & Experiment & SD & p-vaule \\
\hline Sobs & 905.50 & 163.69 & 736.30 & 132.41 & 0.02 \\
Chao & 1081.619 & 157.36 & 911.18 & 138.26 & 0.02 \\
Ace & 1080.67 & 157.61 & 899.67 & 134.84 & 0.02 \\
Shannon & 4.68 & 0.73 & 4.40 & 0.30 & 0.08 \\
Simpson & 0.05 & 0.04 & 0.05 & 0.01 & 0.12 \\
\hline
\end{tabular}



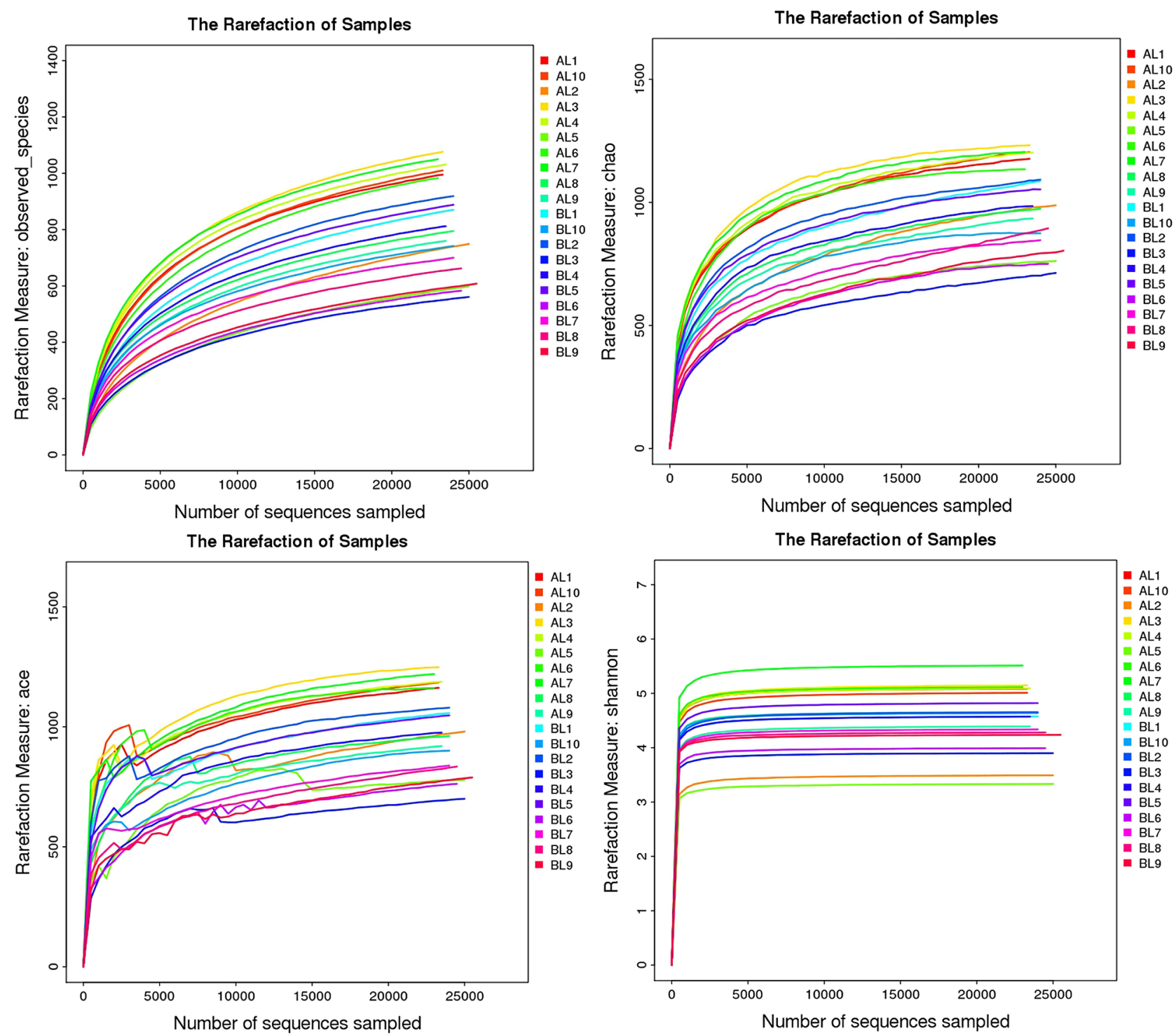

The Rarefaction of Samples

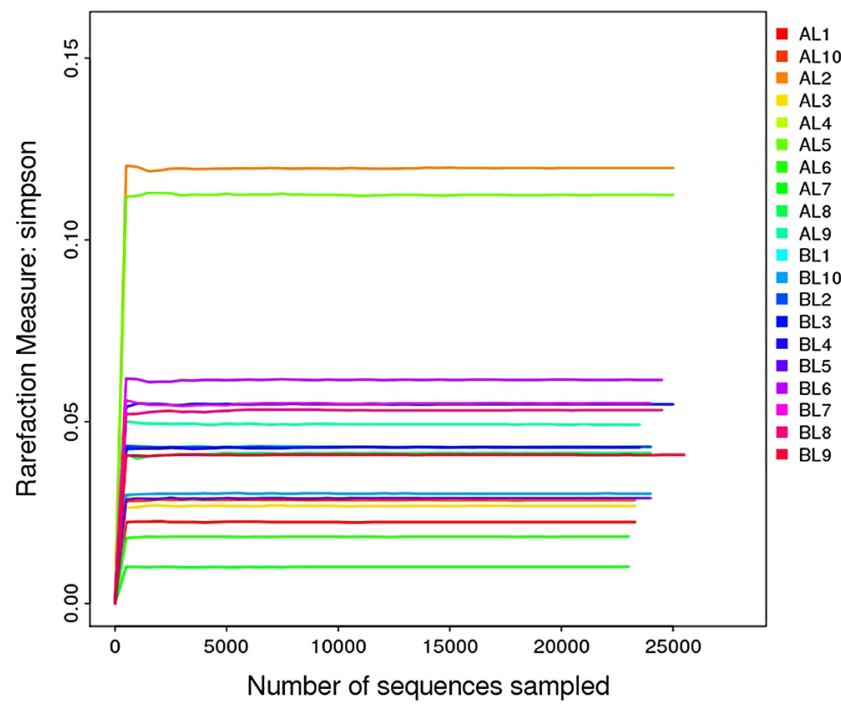

Figure 9. Rarefaction curves of alpha diversity in samples. 
Table 7. Major differential metabolic pathways in perinatal cows.

\begin{tabular}{cc}
\hline Differential metabolic pathway & Annotated metabolite number \\
\hline Tbiquinone and other terpenoids biosynthesis & 17 \\
Tyrosine metabolism & 13 \\
Drug metabolism-cytochrome P450 & 14 \\
Phenylalanine metabolism & 15 \\
Tryptophan metabolism & 26 \\
Neuroactive ligand-receptor interaction & 12 \\
Cytochrome P450 metabolism of foreign compounds & 11 \\
Arachidonic acid metabolism & 17 \\
Steroid hormone biosynthesis & 20 \\
Chemical carcinogenesis & 12 \\
Methyl 2-oxocyclopentanecarboxylate & 17 \\
Alpha-linolenic acid metabolism & 12 \\
Amino acid biosynthesis & 10 \\
\hline
\end{tabular}

statistical analysis, the p-value generated by the statistical test was subjected to FDR correction to obtain a q-value. The final result presents two indicators, the fold change and the q-value, in the form of a Volcano plot. Metabolites with a fold difference $\geq 1.2$ or $\leq 0.8333$, and a q-value $<0.05$ was considered differential. Figure 10 shows the volcano maps in cation and anion modes, respectively.

\subsection{Partial Least Squares Discriminant Analysis}

Partial least squares discriminant analysis (PLS-DA) is a supervised discrimination analysis statistical method that can best reflect the differences between classification groups. The method uses partial least squares regression to establish a relationship model between metabolite expression and sample categories to model and predict sample categories. It can be seen from Figure 11 that the metabolites of the control group and the test group had significant differences.

\subsection{Differential Metabolite Isolation and Identification}

Figure 12 shows a differential ion clustering analysis of cation and anion modes. The screening revealed 639 differential cations, among them, 401 cations were upregulated and 238 were downregulated. A total of 657 differential ions were identified in anion mode, of which 469 were up-regulated and 188 were downregulated.

\subsection{Identification of Differential Metabolic Pathways}

Metabolic pathway analysis can help understanding the major biochemical metabolic pathways and signal transduction pathways in which the metabolites are involved. The annotation of metabolite metabolic pathways in this project was 

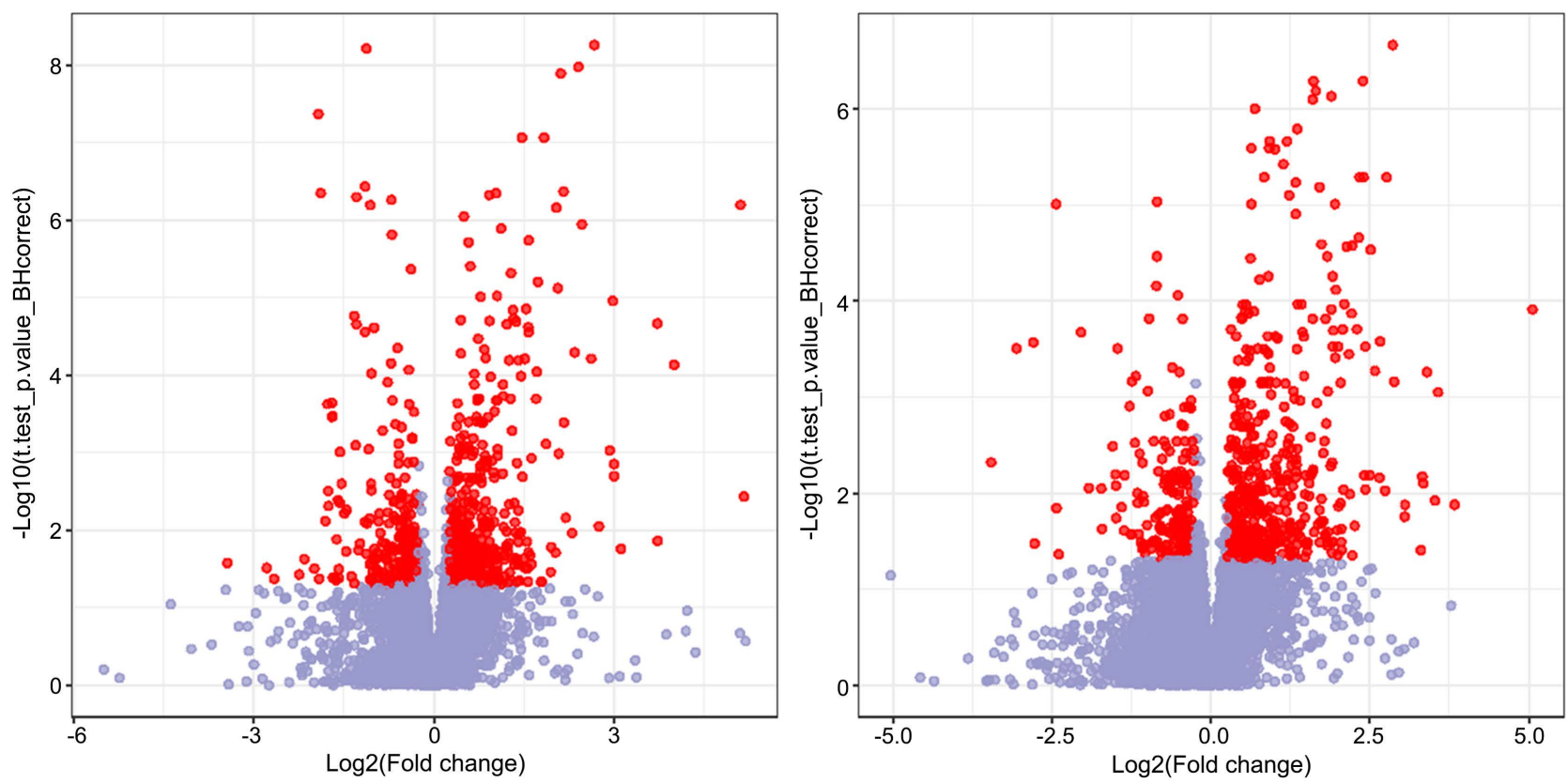

Figure 10. Volcano plot of cations and anions. Note: The abscissa is $\log _{2}$ (fold change), and ordinate is $-\log 10$ (q-value). Data points with fold change smaller than or equal to 0.8333 , or greater than, or equal to 1.2 , and q value $<0.05$ were plot red, while others were blue.
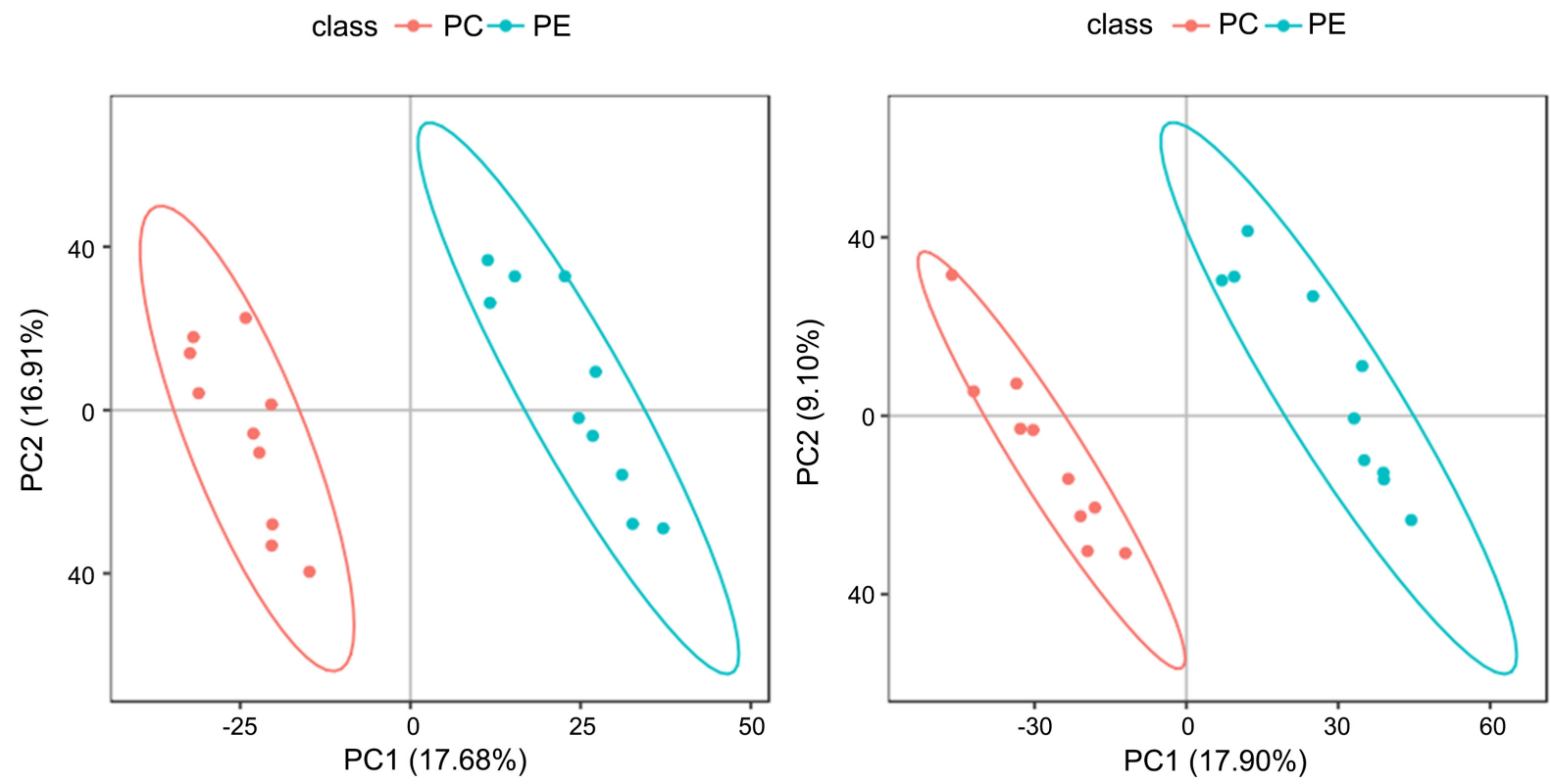

Figure 11. PLS-DA discriminant analysis model in cation mode and anion mode. Note: The abscissa represents the first principal component PC1. The ordinate represents the second principal component PC2. Each point in the figure represents a sample. The degree of dispersion of the two-color symbols represents the distribution trend of the two groups of samples on the PC1 and PC2 axes.

based on the KEGG database. A total of 109 differential metabolic pathways were detected in this experiment. The metabolic pathways with consistent results in cation and anion modes, and annotated number of metabolites greater than 10 were selected as follows. 

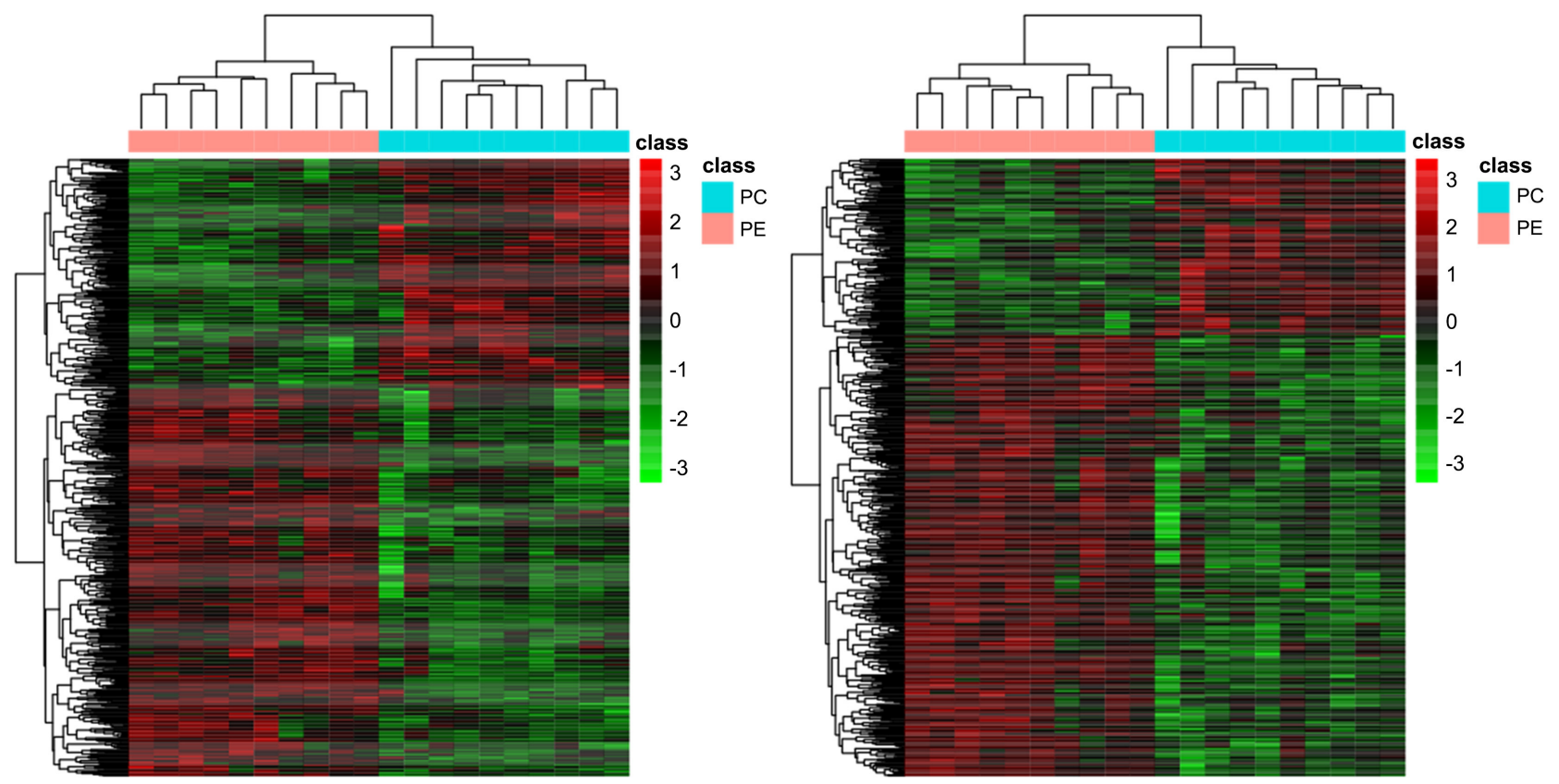

Figure 12. Clustering analysis of differential cations and anions. Note: Each row in the graph represents a differential ion. Each column represents a sample, with colors from green to red indicating intensity from low to high.

\section{Discussion}

Effect of Saccharomyces cerevisiae and Its Fermentation Products on Production Performance

In this experiment, feeding Saccharomyces cerevisiae and its fermentation products had no significant effect on the apparent digestibility of nutrients in postpartum dairy cows. This is contrary to some literature results, but it is consistent with many studies in recent years. Zaworski added yeast fermentation to the ration of perinatal dairy cows and found that the amount of yeast fermentation added had no significant effect on the examined indexes [9]. In our test, compared with the control group, the test group increased milk yield by $5.2 \pm 2.3$ $\mathrm{kg} / \mathrm{d}$, significantly increased the milk protein and lactose content, and reduced the number of somatic cells in the milk, and reduced the postpartum insulin level. Addition of probiotics had no significant effect on the digestibility of nutrients. After supplementation with $S$. cerevisiae and its fermentation products, the DMI in postpartum dairy cows was significantly improved. This is consistent with the findings of Dann and Yuan [10]. However, studies by Arambel, Swartz, and Robinson have shown that yeast does not affect feed intake in peripartum dairy cows [11] [12] [13].

Zaworski argued that the unique aroma of yeast ferment is an important factor in promoting dairy feeding, especially when the feed quality is low and the appetite is low [9]. Another possibility is that yeast fermentation can ameliorate the stress caused by dietary changes. This explains why there is no significant change in feed intake in pre-lactation cows and the different outcomes of similar trials. Because whether yeast fermentation can promote feed intake is also re- 
lated to feed quality and individual differences. There is no final conclusion about the mechanism of action of yeast ferment on dry matter intake, and more data support is needed. Feeding $S$. cerevisiae and its fermentation products can significantly increase the lactose content in milk of peripartum dairy cows after delivery, and tends to increase milk production, but has no significant effect on other milk components. The crude fat digestibility in the experimental group was increased, and the digestibility of other nutrients did not differ significantly. This also shows that yeast can promote the body's energy utilization. Supplemental feeding of Saccharomyces cerevisiae and its fermentation products can significantly reduce milk protein content $(\mathrm{P}<0.01)$. After supplementation with Saccharomyces cerevisiae and its fermentation products, the number of somatic cells in the milk decreased, but the statistical results were not significant $(\mathrm{P}=$ 0.75). This may be due to a large difference within the group. There is no significant effect on other milk components.

Effect of Saccharomyces cerevisiae and Its Fermentation Products on Serum Hormone Level

Supplementation with Saccharomyces cerevisiae and its fermentation products can increase glucagon, and $\beta$-hydroxybutyrate content and reduce insulin content in dairy cows. At the same time, the supplemental Saccharomyces cerevisiae and its fermentation products also reduced the free fatty acid content in lactating cows and decreased the leptin content. Glucagon and insulin antagonize each other, regulate the balance of glucose metabolism in animals, and thus affect energy metabolism. As a source of $\beta$-hydroxybutyric acid, the butyric acid produced by rumen fermentation is transformed by the rumen epithelium. However, some previous studies have shown that yeast does not increase ruminal butyric acid content, regardless it is in vivo [14] or in vitro [13], which is inconsistent with the results of this study. Another important source of beta-hydroxybutyrate is the ketogenic action of the liver during pre-lactation. To meet the energy needs of pregnancy and lactation, cows need to mobilize large amounts of triglycerides from adipose tissue and flow into the blood in the form of glycerol and free fatty acids [1] and participate in gluconeogenesis in the liver or to regenerate triglycerides. Therefore, free fatty acids in the blood reflect the intensity of lipid mobilization. Although it is an important source of energy and a precursor to milk fat, too much free fatty acids will increase the burden on the liver. Beta oxidation of free fatty acids can produce acetyl-CoA. Oxidation of acetyl-CoA is restricted during pre-lactation, as both gluconeogenesis and the Krebs cycle compete for oxaloacetate in mitochondria [15]. One way in which free fatty acids are produced is the incomplete oxidation of fatty acids. It can be used for functionality. However, when the concentration is too high, free fatty acids affect immune and metabolic functions and increases the risk of infection and metabolic disorders [16].

As shown in the OTU venn diagram, a total of 1726 OUTs were detected by the two groups. There were 1639 OTUs in the control group, of which 293 OTUs were unique to the control group. The test group had a total of 1433 OTUs, of 
which 87 OTUs were unique to the test group. A total of 1346 OTUs are common to both treatment groups. It is generally believed that an OTU corresponds to a species that actually exists in nature. Through the analysis of principal components and Alpha diversity, we can also get the same conclusion. Supplementation of probiotics during perinatal period can significantly reduce the abundance of rumen microbes in dairy cows, but has no significant effect on microbial diversity. Jami reported that the abundance of a large number of rumen bacterial species is highly correlated with lactation production or feeding efficiency [4]. This means that bacterial communities play an important role in regulating host physiological parameters. Some studies have suggested that the addition of yeast has an effect on the abundance of fibrinolytic bacteria [17] and has an effect on lactic acid-utilizing bacteria [18] [19]. However, no one has studied the association between unidentified bacterial abundance, known bacterial abundance and production performance when supplementing Saccharomyces cerevisiae [20].

Among the solid and liquid components of the rumen contents, Prevotella is the most abundant bacteria, accounting for approximately $42 \%$ to $60 \%$ of the total bacterial population [20]. Current research suggests that Prevotella has a negative correlation with dry matter intake and milk fat percentage [4]. They have nothing to do with dose and viability, and yeast supplements have no effect on Prevotella, whether solid or liquid [22]. In the solid phase rumen contents and liquid phase rumen contents, the relative abundance of the genus Bacillus was positively correlated with the milk fat percentage and dry matter intake. This reflects the presence of $F$. succinogenes, the major fiber-decomposing bacterium in the rumen [21]. However, we do not know why the relative abundance of fibrin in the solid phase rumen contents was negatively correlated with milk production. In the solid phase and the liquid phase, the ratio of the genus Fibrin to the milk protein was positively correlated. In particular, yeast does not alter the relative abundance of the genus Fibrin. In the rumen solid phase content, the relative abundance of rumen cocci is positively correlated with the degradation rate of acid detergent fiber. The rumen liquid content was positively correlated with dry matter intake and milk fat percentage. This may be due to the decomposition of fibers by $R$. albus and $R$. flavefaciens.

\section{Conclusion}

Supplemental feeding of Saccharomyces cerevisiae and its fermentation products can significantly increase the feed intake of peripartum dairy cows, the milk production increased by $5.2 \% \pm 2.3 \mathrm{~kg} / \mathrm{d}$, and the number of somatic cells decreased. In addition, it can increase postpartum lactose content, increase blood levels of glucagon and BHBA, and increase leptin content during early lactation, but it significantly reduced the insulin content. Supplemental feeding of Saccharomyces cerevisiae and its fermentation products can significantly increase the content of acid and isovaleric acid in the rumen fluid of perinatal dairy cows and reduce the 
content of valeric acid. Reduced rumen microbial abundance at 21 days postpartum, but had no effect on microbial diversity. At the same time, the content of the bacillus was significantly increased while the content of the fungus and Candidatus_Saccharibacteria decreased.

\section{Acknowledgements}

The study was financially supported by the earmarked fund for the Key R \& D plan of shandong province (2019JZZY010704) and thanks for the cooperation with Shandong Bilan Biological Technology Company. It was also supported by the National Key R \& D program of China (2017YFD0500502), Cattle Agro-industry Technology Research System of Shandong Province (SDAIT-12-011-06), Natural Science Fund of China (31572427) (31372340) and Taishan scholar project.

\section{Conflicts of Interest}

The authors declare no conflicts of interest regarding the publication of this paper.

\section{References}

[1] Drackley, J.K. (1999) Biology of Dairy Cows during the Transition Period: The Final Frontier? Journal of Dairy Science, 82, 2259-2273. https://doi.org/10.3168/jds.S0022-0302(99)75474-3

[2] Hernandezsanabria, E., Goonewardene, L.A., Wang, Z., Durunna, O.N., Moore, S.S. and Guan, L.L. (2012) Impact of Feed Efficiency and Diet on Adaptive Variations in the Bacterial Community in the Rumen Fluid of Cattle. Applied \& Environmental Microbiology, 78, 1203-1214. https://doi.org/10.3168/jds.S0022-0302(99)75474-3

[3] Carberry, C.A., Kenny, D.A., Han, S., Mccabe, M.S. and Waters, S.M. (2012) Effect of Phenotypic Residual Feed Intake and Dietary Forage Content on the Rumen $\mathrm{Mi}$ crobial Community of Beef Cattle. Applied \& Environmental Microbiology, 78, 4949 4958. https://doi.org/10.1128/AEM.07759-11

[4] Jami, E., White, B.A. and Mizrahi, I. (2014) Potential Role of the Bovine Rumen Microbiome in Modulating Milk Composition and Feed Efficiency. PLoS ONE, 9, e85423. https://doi.org/10.1371/journal.pone.0085423

[5] Oh, J., Harper, M., Melgar, A., Compart, D.M.P. and Hristov, A.N. (2019) Effects of Saccharomyces cerevisiae-Based Direct-Fed Microbial and Exogenous Enzyme Products on Enteric Methane Emission and Productivity in Lactating Dairy Cows. Journal of Dairy Science, 102, 6065-6075. https://doi.org/10.3168/jds.2018-15753

[6] Fomenky, B.E., Do, D.N., Talbot, G., Chiquette, J., Bissonnette, N., Chouinard, Y.P., Lessard, M. and Ibeagha-Awemu, E.M. (2018) Direct-Fed Microbial Supplementation Influences the Bacteria Community Composition of the Gastrointestinal Tract of Pre- and Post-Weaned Calves. Scientific Reports, 8, Article No. 14147. https://doi.org/10.1038/s41598-018-32375-5

[7] Ramsing, E.M., Davidson, J.A., French, P.D., Yoon, I., Keller, M., Peters-Fleckenstein, H., et al. (2009) Effects of Yeast Culture on Peripartum Intake and Milk Production of Primiparous and Multiparous Holstein Cows. Professional Animal Scientist, 25, 487-495. https://doi.org/10.3168/jds.2013-7692

[8] Zaworski, E.M., Shrivermunsch, C.M., Fadden, N.A., Sanchez, W.K., Yoon, I. and 
Bobe, G. (2014) Effects of Feeding Various Dosages of Saccharomyces cerevisiae Fermentation Product in Transition Dairy Cows. Journal of Dairy Science, 97, 30813098. https://doi.org/10.3168/jds.2013-7692

[9] Dann, H.M., Drackley, J.K., Mccoy, G.C., Hutjens, M.F. and Garrett, J.E. (2000) Effects of Yeast Culture (saccharomyces cerevisiae) on Prepartum Intake and Postpartum Intake and Milk Production of Jersey Cows. Journal of Dairy Science, 83, 123-127. https://doi.org/10.3168/jds.S0022-0302(00)74863-6

[10] Arambel, M.J. and Kent, B.A. (1990) Effect of Yeast Culture on Nutrient Digestibility and Milk Yield Response in Early- to Midlactation Dairy Cows. Journal of Dairy Science, 73, 1560-153. https://doi.org/10.3168/jds.S0022-0302(90)78825-X

[11] Swartz, D.L., Muller, L.D., Rogers, G.W. and Varga, G.A. (1994) Effect of Yeast Cultures on Performance of Lactating Dairy Cows: A Field Study. Journal of Dairy Science, 77, 3073-3080. https://doi.org/10.3168/jds.S0022-0302(94)77249-0

[12] Robinson, P.H. and Garrett, J.E. (1999) Effect of Yeast Culture (Saccharomyces cerevisiae) on Adaptation of Cows to Postpartum Diets and on Lactational Performance. Journal of Animal Science, 77, 988-999. https://doi.org/10.2527/1999.774988x

[13] Sullivan, H.M. and Martin, S.A. (1999) Effects of a Saccharomyces cerevisiae, Culture on in Vitro Mixed Ruminal Microorganism Fermentation. Journal of Dairy Science 82, 2011-2016. https://doi.org/10.3168/jds.S0022-0302(99)75438-X

[14] Harrison, G.A., Hemken, R.W., Dawson, K.A., Harmon, R.J. and Barker, K.B. (1988) Influence of Addition of Yeast Culture Supplement to Diets of Lactating Cows on Ruminal Fermentation and Microbial Populations. Journal of Dairy Science, 71, 2967-2975. https://doi.org/10.3168/jds.S0022-0302(88)79894-X

[15] Bobe, G., Velez, J.C., Beitz, D.C. and Donkin, S.S. (2009) Glucagon Increases Hepatic mRNA Concentrations of Ureagenic and Gluconeogenic Enzymes in EarlyLactation Dairy Cows. Journal of Dairy Science, 92, 5092-5099. https://doi.org/10.3168/jds.2009-2152

[16] Ingvartsen, K.L. and Moyes, K. (2013) Nutrition, Immune Function and Health of Dairy Cattle. Animal, 7, 112-122. https://doi.org/10.1017/S175173111200170X

[17] Callaway, E.S. and Martin, S.A. (1997) Effects of a Saccharomyces cerevisiae Culture on Ruminal Bacteria That Utilize Lactate and Digest Cellulose. Journal of Dairy Science, 80, 2035-2044. https://doi.org/10.3168/jds.S0022-0302(97)76148-4

[18] Chaucheyras, F., Fonty, G., Bertin, G., Salmon, J.M. and Gouet, P. (1996) Effects of a Strain of Saccharomyces cerevisiae (Levucell ${ }^{\circledR}$ SC), a Microbial Additive for Ruminants, on Lactate Metabolism in Vitro. Canadian Journal of Microbiology, 42, 927-933. https://doi.org/10.1139/m96-119

[19] Rossi, F., Luccia, A.D., Vincenti, D. and Cocconcelli, P.S. (2016) Effects of Peptidic Fractions from Saccharomyces cerevisiae Culture on Growth and Metabolism of the Ruminal Bacteria Megasphaera elsdenii. Animal Research, 53, 177-186. https://doi.org/10.1051/animres:2004009

[20] Jiang, Y., Ogunade, I.M., Arriola, K.G., Qi, M., Vyas, D. and Staples, C.R., et al. (2017) Effects of the Dose and Viability of Saccharomyces cerevisiae. Ruminal Fermentation, Performance of Lactating Dairy Cows, and Correlations between Ruminal Bacteria Abundance and Performance Measures. Journal of Dairy Science, 100, 8102-8118. https://doi.org/10.3168/jds.2016-12371

[21] Cato, E.P., Moore, W.E.C. and Bryant, M.P. (1978) Designation of Neotype Strains for Bacteroides Amylophihs Hamlin and Hungate 1956 and Bacteroides Succinogenes Hungate 1950. International Journal of Systematic Bacteriology, 28, 491-495. 
https://doi.org/10.1099/00207713-28-4-491

[22] Jiang, Y., Ogunade, I.M., Qi, S., Hackmann, T.J., Staples, C. and Adesogan, A.T. (2017) Effects of the Dose and Viability of Saccharomyces cerevisiae. 2. Diversity of Ruminal Microbes as Analyzed by Illumina miseq Sequencing and Quantitative PCR. Journal of Dairy Science, 100, 325-342. https://doi.org/10.3168/jds.2016-11263 